\title{
Methods of the Long-term Radial-Velocity Variation Removal and their Application to Detect Duplicity of Several Be Stars
}

\author{
J. Nemravová ${ }^{1}$, P. Harmanec ${ }^{1}$, P. Koubský ${ }^{2}$ and A. Miroshnichenko ${ }^{3}$ \\ ${ }^{1}$ Astronomical Institute of the Charles University, Faculty of Mathematics and Physics, \\ V Holešovičkách 2, CZ-180 00 Praha 8, Czech Republic \\ email: janicka.ari@seznam.cz, hec@sirrah.troja.mff.cuni.cz \\ ${ }^{2}$ Astronomical Institute of the Academy of Sciences, CZ-251 65 Ondřejov, Czech Republic \\ email: koubsky@sunstel.asu.cas.cz \\ ${ }^{3}$ Department of Physics and Astronomy, University of North Carolina at Greensboro, \\ Greensboro, NC 27402, USA \\ email: a_mirosh@uncg.edu
}

\begin{abstract}
There are several types of binary stars which show non-periodical radial velocity variations with the amplitude larger than those connected with the orbital motion. The nonperiodical changes have to be removed in order to study the orbital ones. We propose three removal techniques, two of which are based on the trend modeling with continuous functions and the third one that takes the orbital motion into account.
\end{abstract}

Keywords. methods: data analysis, techniques: radial velocities, stars: emission-line, Be

\section{Introduction}

The detection of duplicity of Be stars might be very complicated. Low-amplitude radialvelocity (RV hereafter) orbital changes can be masked by cyclic variations related to changes and asymmetries in the circumstellar matter surrounding such stars. If one wants to study the orbital motion of a Be binary, these cyclic variations have to be removed. We present three techniques that are suitable to the task.

\section{Methods}

(a) The first method is optimal in situations when one has little idea about the value of the orbital period. It is based on a smoothing technique via spline functions developed by Vondrák $(1969,1977)$. To its practical application, we used the program HEC13, which allows us to smooth either individual observations or normal points averaged over constant time intervals. Cyclic variations modelled with the method are shown in the first panel of Fig. 1.

(b) The second method comes in order when one knows the true orbital period. One has to split the RVs into subsets, each being several times shorter than the typical timescale of the long-term changes. Each subset should be as short as possible to obtain the resolution of the long-term changes possible, but it must contain data reasonably defining the orbital RV curve. An individual systemic velocity is then derived for each subset as part of the orbital solution with the SPEL program. This way, the long-term trend is removed in discrete time steps. The resulting ' $\gamma$ velocities' are shown with empty squares in the first and the third panels of Fig. 1. 

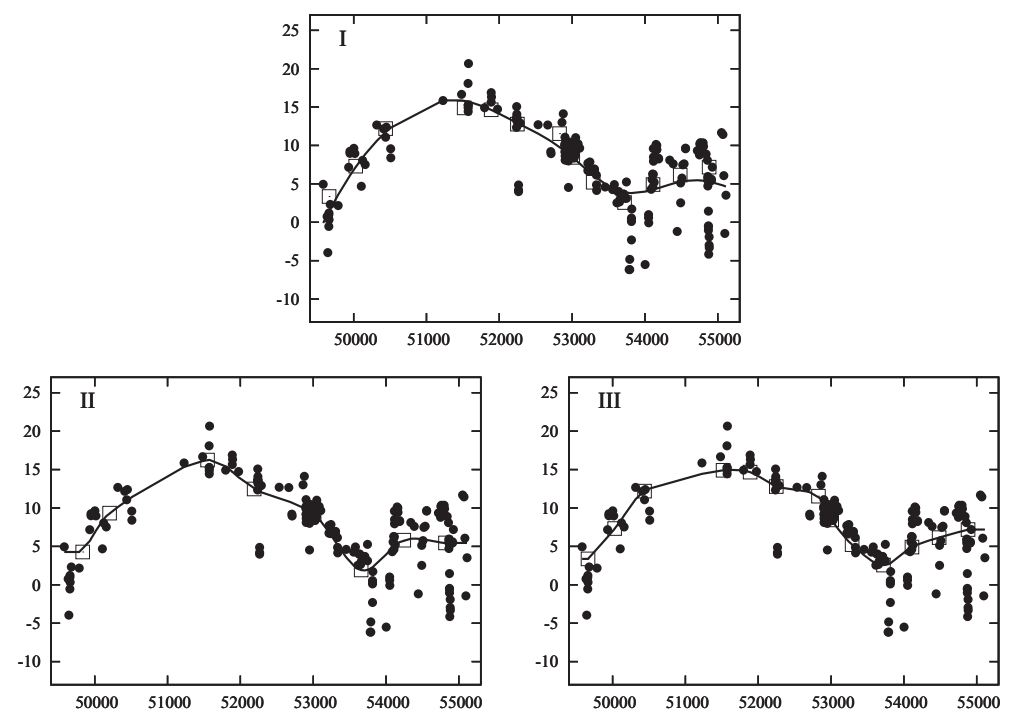

Figure 1. RVs measured on the steep wings of $\mathrm{H} \alpha$ line of BU Tauri. The solid line represents long-term variations estimated with: HEC13 (panel I) and HEC36 (panels II and III). Empty squares represent: $\gamma$ velocities computed with SPEL (panels I and III) and RVs averaged over constant time interval (panel II).

(c) The third method models the long-term changes with Hermite polynomials through suitably chosen normal points. The program HEC36, designed for this task, employs the interpolation subroutine INTEP (see Hill 1982). If one chooses to input normal points computed over constant time intervals, the results are very similar to the ones obtained with the first method. We found that it is better to use the program HEC36 to improve the second method. This can be done by submitting the $\gamma$ velocities computed with SPEL as the normal points to the program HEC36. The trend estimated with the normal points computed over constant time intervals is shown in panel II and with velocities taken as normal points in panel III of Fig. 1.

\section{Comparison}

The second method resulted in an orbital RV curve with significantly lower rms for BU Tauri (Nemravová et al. 2010) and $\gamma$ Cassiopeiae (in preparation) than smoothing with the first method. The main reason is that the systemic velocities computed with SPEL properly model the orbital RV curve of the given subset. This does not mean that the second method must always be superior to the first one. We recommend trying all three methods in every particular study and comparing the results. HEC13 and HEC36 written by PH are available for download at http://astro.troja.mff.cuni.cz/ $\mathrm{fpt} / \mathrm{hec} /$.

\section{Acknowledgements}

The research of JN and PH was supported by the grant P209/10/0715 of the Czech Science Foundation and by the grant SVV-263301 of the Charles University of Prague.

\section{References}

Hill G. 1982, Publ. Dominion Astrophys. Obs. Victoria, 16, 67

Nemravová, J., Harmanec, P.,Kubát, J., Koubský, P., Iliev, L., Yang, S., Ribeiro, J., Šlechta, M., Kotková, L., Wolf, M., \& Škoda, P. 2010, Aछ̛AS, 516, A80

Vondrák J. 1969, Bull. Astron. Inst. Czechosl., 20, 349

Vondrák J. 1977, Bull. Astron. Inst. Czechosl., 28, 84 